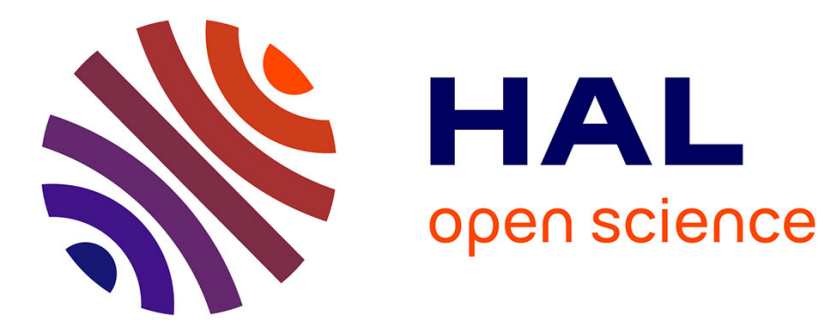

\title{
Graph theory
}

César Ducruet

\section{To cite this version:}

César Ducruet. Graph theory. International Encyclopedia of Geography: People, the Earth, Environment and Technology, 85, John Wiley \& Sons, Ltd, pp.102729, 2016, 10.1002/9781118786352.wbieg0490 . halshs-02610623

\section{HAL Id: halshs-02610623 https://shs.hal.science/halshs-02610623}

Submitted on 9 Dec 2020

HAL is a multi-disciplinary open access archive for the deposit and dissemination of scientific research documents, whether they are published or not. The documents may come from teaching and research institutions in France or abroad, or from public or private research centers.
L'archive ouverte pluridisciplinaire HAL, est destinée au dépôt et à la diffusion de documents scientifiques de niveau recherche, publiés ou non, émanant des établissements d'enseignement et de recherche français ou étrangers, des laboratoires publics ou privés. 


\title{
Graph Theory
}

\author{
Dr. César Ducruet \\ Centre National de la Recherche Scientifique (CNRS) \\ cdu@parisgeo.cnrs.fr
}

Pre-final version of the entry published in Richardson D. et al. (Eds.) (2017) The International Encyclopedia of Geography, Wiley-Blackwell \& Association of American Geographers, https://doi.org/10.1002/9781118786352.wbieg0490

\begin{abstract}
Originally a branch of mathematics designed to formalize the topological properties of networks, graph theory has evolved dramatically in the last two decades through interdisciplinary research encompassing both social and natural scientists. Geographers, who have been actively pushing for the integration of graph theoretical methods into geography in the 1960s have somewhat neglected recent advances in network theory and analysis originating from graph theory.
\end{abstract}

The origins of graph theory are often attributed to the works of Leonhard Euler. In a classic study of the city of Koenigsberg (1735), he designed a problem where a set of nodes (or vertices) connected by links (or edges), had to be traveled without passing two times by the same road or bridge. Such an exercise proved to be mathematically impossible, and motivated further efforts to define the topological properties of graphs during the subsequent decades and centuries. Still nowadays, the application of graph theory to geography remains largely focused on technical networks, using traditional mathematical tools (Arlinghaus et al., 2002). This entry describes graph theory in general as well as its use by geographers, notwithstanding parallel evolutions of the field in other social and natural sciences, and their mutual influences.

A wide and specific vocabulary describes various sorts of graphs having distinct internal structures, as well as subgraphs (subset of connected nodes) and individual nodes by the architecture of their mutual links. Graphs may be visualized either through matrices or by the node/link view, and many graph visualization softwares exist nowadays, capable of applying numerous types of visualizations and measures even on very large networks composed of 
thousands of links and nodes. Visualizing large graphs is often an exercise with limited outputs, due to poor readability, so that many different operations of network simplification should be applied prior to visualization, such as filtering links or nodes according to certain quantitative thresholds or qualities. Furthermore, visualizing a network on a map, such as in geography (but this has become so popular nowadays that any spatially embedded dataset made of nodes and links can be instantaneously projected on the screen regardless of its size etc.), brings in additional issues of readability. The matrix itself (or adjacency matrix) is represented by a table where nodes are rows and columns, and links are, in their simplest understanding, represented in a binary fashion by the number 0 or 1 where lines and columns intersect. There are specific mathematical calculations based on matrices that we do not detail in this review. Visualizing graphs as sets of nodes connected by links proved to be more useful to illustrate the existence of various network morphologies, or fundamental components of networks, such as cycles, cliques, chains, motifs, but also various situations characterizing individual nodes, such as isthmus, bridge, ego network, star, etc. Graph theory is based on a specific vocabulary to describe networks. Nodes and links are labelled vertices (vertex) and edges, respectively. Vertices may be junctions or end points in the graph, with edges between them. Connexity refers to the property of a graph to have all its vertices interconnected, which otherwise contains isolates (i.e. vertices without edges) or at least two connected components. A complete graph is defined by the maximum number of edges among vertices. The graph is directed (digraph) when two edges exist between two same vertices, referring to two directions (AB vs. BA). When both directions exist the graph is said symmetrical, otherwise asymmetrical. Other dimensions are also important, such as when vertices and edges have certain values, in a weighted graph (e.g. passenger flow, number of vehicles); when vertices are connected by only one type edges 
(simple graph) or at least two types (multigraph, multiplex graph), such as, in a social network, friendship and professional ties. Finally, a bipartite graph exists when two or more types of vertices are connected, such as conferences and speakers, journals and scholars, etc. at the condition that vertices of the same nature are not connected with each other.

Numerous internal structures have also been identified within graphs at different levels of their organization. For instance, chains and cycles are both continuous alignment of edges, while the cycle starts and ends at the same vertex. A path between two nodes is a sequence of edges travelled in the same direction. Several paths may exist between two same nodes, thus motivating the search for the shortest path and of the minimum cost path, the latter being only possible in the case of a weighted graph where edges are valued by a distance, time, or other metric (e.g. days, hours, kilometers, monetary value). For the shortest path, topological distance refers to the number of edges to be crossed in order to reach the final destination of the path. Topological distance is often referred to as path (or edge) length but also depth. The clique is one fundamental structure of graph theory, defined as a maximal complete subgraph, i.e. a subset of nodes being fully connected with each other, the smallest example being the triangle of " 3 clique". In social network analysis (SNA), the clique is an essential component of social organization, and is often opposed to the "star" configuration where several vertices are connected to only one vertex. The ego network comprises the connected neighbors of a given vertex as well as the edges among these neighbors. More complex internal structures have been the focus of several researches on graphs, such as the so-called "clusters" or "communities", which definition may vary according to several criteria. Overall, a cluster or community is defined either by a higher density of connections or by the similarity of their connections. In other words, tightly connected vertices may form a community with a high probability to be 
relatively close to each other in the graph. Vertices having similar connectivity to others, conversely, may be close or far away to each other. Vertices and edges lying in between two or more communities are often labelled isthmuses or bridges, due to their high importance. In social network analysis, it is also called weak tie or structural hole in the case of edges in-between different groups. Finally, a graph is said assortative when the number of edges of a given vertex is positively and significantly correlated with the average number of edges of its direct neighbors in the graph, otherwise it is said disassortative.

Based on the aforementioned characteristics, graphs may be labelled differently in terms of their overall structural properties. The regular graph (or k-regular) possesses exactly the same number of edges per vertex. The random graph exhibits a normal or Gaussian statistical distribution of the number of edges per vertex. Scale-free and small-world graphs are defined below when it comes to the more recent field of complex networks. The tree graph refers to a situation where no clique, cluster, or cycle can be found, as in the case of river networks for instance, which function as dendrograms. More conventional measures help revealing the overall structure of the graph and define its main character. For instance, the diameter is the length of the longest of the shortest paths among all pairs of vertices in the graph, measured by the number of edges needed to be travelled. The average shortest path length is similar but refers to the average number of edges composing the shortest paths across the graph, while high values often imply more difficulty to circulate in the network. Planar graphs often have high values for both diameter and average shortest path length. The average clustering coefficient (or transitivity) measures on average the proportion of closed triangles in the maximum possible number of triangles in the graph. In that sense it is close to another classic measure of graph theory, the alpha index, which looks at the proportion of existing cycles in the maximum possible number of 
cycles, with the difference that a cycle can have a length of three edges (triangle, 3-clique) but also of four, five, etc. High values of the average clustering coefficient mean that the graph is tightly connected, while tree graphs will always have zero.

One of the first imports from mathematics into geography was made by Kansky (1963), incorporating previous studies on telecommunication flows and highway networks among US cities. Such works notably reviewed the various measures and indices applicable to mostly planar graphs, i.e. graphs where two edges cannot cross without creating a vertex, as it is often the case for road, rail, and river networks; the main goal being to describe the morphology, connectivity, and density of various graphs (Haggett and Chorley, 1969). Without reviewing them entirely, most common indices include the diameter (number of links between the two most distant nodes), the alpha (share of observed cycles in the total possible number of cycles - the cycle being a closed path of various length in terms of its number of links), beta (number of links per node, or density), and gamma (share of observed links in the total possible number of links, or completeness) indices. Three limitations of such general indices should be addressed, however. First, one major drawback is that two networks of identical size (number of nodes and links) but with drastically different topology (e.g. star-shaped network where one node connects all others vs. chain-like network formed by an alignment of links) will be characterized by same results. Second and related with the first, general indices are not robust to network size, so that their level will vary according to the size of the network. Third, graph theory and its application to geography long remained binary (presence or absence of a link between two nodes), thereby completely ignoring the possible weight of links (e.g. traffic intensity or capacity, kilometric length) and their quality (e.g. links and nodes of different types). 
Other measures include those at the local level, such as calculating, for each node, its farness to all other nodes by the number of links needed to connect the most distant node (Koenig number) or the total length of shortest paths connecting all other nodes (Shimbel accessibility index) (Figure 1). Such studies focusing on small, planar networks thus largely neglected the analysis of non-planar networks (i.e. allowing edge crossings without creating nodes). Due to limited interactions with Social Network Analysis (SNA) that developed from the 1920s onwards, no imports have been realized from sociology although the latter had developed many tools for analyzing non-planar networks. For instance, betweenness centrality (number of times a node lies on a shortest path in the graph) as well as transitivity (or clustering coefficient) came from SNA, and later on were integrated in studies of complex networks. They consider that for each node, there is a share of observed triangles - or cliques - in the maximum possible number of triangles among its neighbors. Transitivity had no use for studying planar networks since rectangles rather than triangles are often found in road or railway networks. It is only in the early 1990s that geographers started to analyze networks of different nature, or non-planar networks, such as those formed by multinational corporations, airlines, and shipping lines, thereby stepping out of classic graph theory (Ducruet and Beauguitte, 2014) and from a more urban perspective. Other important refinements of graph theory have been to consider the weight and spatial dimension of networks. The cited measures have been adapted to incorporate the weight of links and nodes so as to better reflect inequality in, for instance, traffic distribution, compared with the sole topological (binary) aspects. Thus, all measures can be weighted according to various metrics at global (whole network) and local (individual nodes) levels. When it comes to the spatial dimension of networks, physicists were keen to demonstrate the influence of spatial constraints on network growth and topology, whereas such aspects were often taken for 
granted by geographers, since all their studies focused on spatially embedded networks in contrast with studies of non-spatial networks such as social networks, collaborative and citations networks, the world wide web, etc. Geographers focused more on detailed analyses of binary graphs in abstract spaces and of real networks using Geographical Information Systems but often without reference to graph theory. Physicists studying spatial networks notably underlined that preferential attachment occurs over shorter distances, since there is a cost of creating new links to overcome the friction of space, thus resulting in higher average clustering coefficients than in non-spatial networks (Barthelemy, 2011).

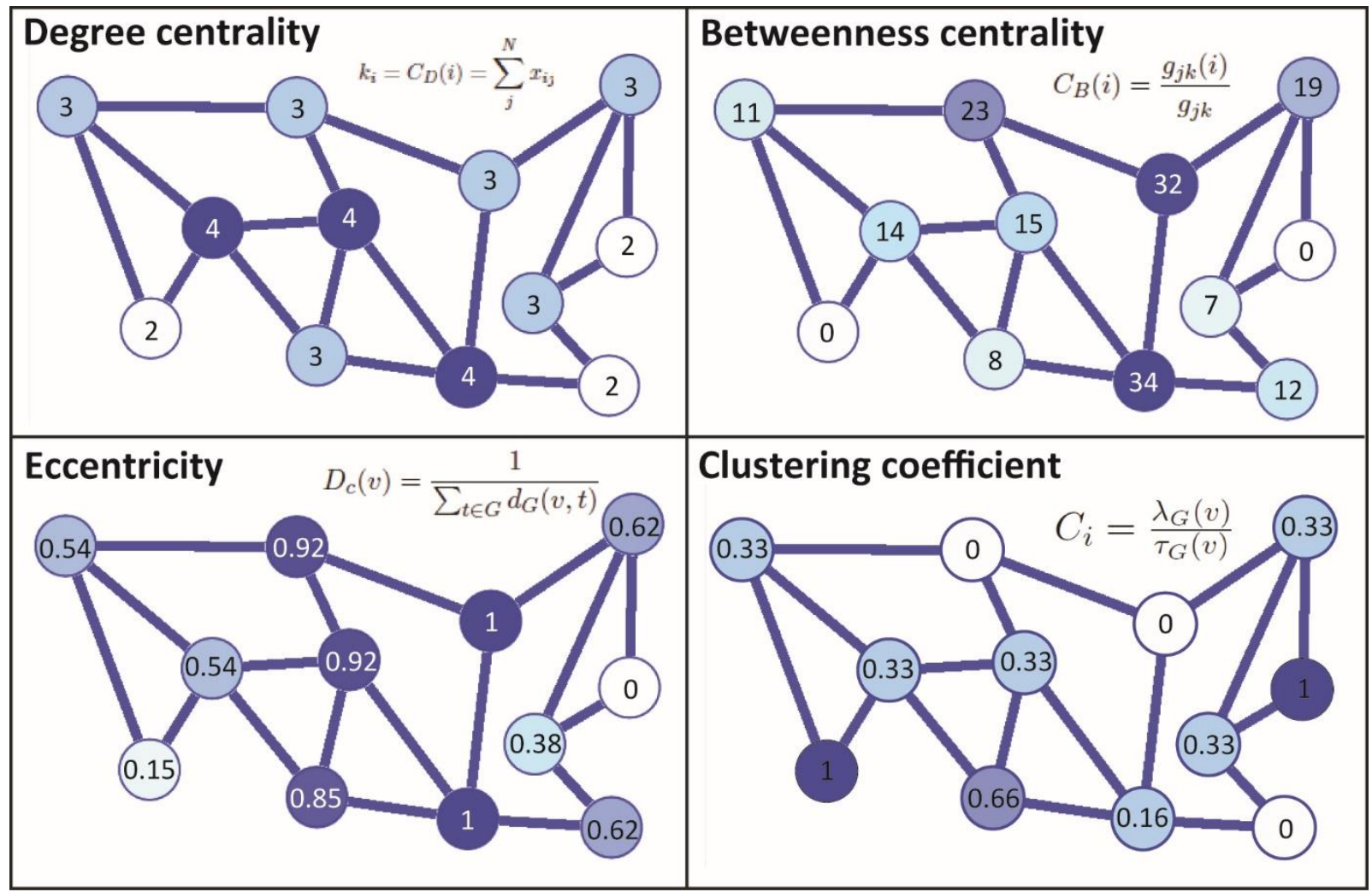

Figure 1: Selected graph-theoretical measures 
Efforts made by geographers to improve their use of graph-theoretical methods occurred in relative isolation, though, from the rapid evolution which graph theory went through in the late 1990s and early 2000s. Natural scientists, such as physicists, have been eager to rejuvenate graph theory and notably the aforementioned limitations as well as its approach of graph dynamics. They notably proposed to define a so-called "complex network" by non-trivial topological attributes, which are neither random nor regular, but are found in most real-world networks. Another dimension of complex networks is their large number of nodes and links, and their analysis clearly benefited from the growing availability of large datasets and computation power. New measures were proposed, although they were in fact a rediscovery of existing ones, such as transitivity (clustering coefficient) originating from SNA and the gamma index (density) proposed by Kansky (1963). Still, the merit of natural scientists has been to provide clearer definitions of network models and their evolutionary mechanisms, with new applications on realworld networks that were traditionally analyzed by social sciences in more limited ways. One of their major achievement has been to complement the random graph model, which were dominant until then (a random graph is characterized by a normal or Gaussian distribution of the nodes' degree), by two new models: scale-free and small-world. The small-world network is defined by a higher average clustering coefficient and smaller diameter than the random graph of equal size (Watts and Strogatz, 1998). It means that small-world networks are more likely to contain cliques (subgroups of at least three connected neighbors forming a closed path) and to provide more efficient ways to circulate in the network, in terms of the length needed to connect its distant parts. Scale-free networks are similar to small-world networks but their distribution of degrees follows a power-law (the slope exponent ranging between 2 and 3), which means that very few nodes have plentiful connections, and a vast majority of nodes have very few 
connections. Another dimension of scale-free networks is the process of preferential attachment by which newly added nodes primarily connect larger nodes, thereby reinforcing the hierarchical structure of the network (Barabasi and Albert, 1999). Despite the spread of such models across almost all scientific disciplines, geography has remained somewhat reluctant in adopting and testing these innovations. In fact, geographers doing network analysis have continuously relied on the same indices and measures over the last five decades. Just like the innovative character of complex network research is questioned, the benefits of applying such methods to test geographic questions also remain unclear. Should this be indebted to the models or to the way they were applied? One part of the answer lies in the fact that geography as a whole went through a "behavioral" turn after the so-called quantitative revolution of the 1960s, graph theory and network analysis - and spatial analysis as a whole - remaining "somewhat of a backwater" since then (Waters, 2006). Networks continued to be analyzed by regional scientists but not necessarily using graph theory, so in even more abstract spaces while focusing on costs, and engineers focusing on performance and optimization. Yet, a small number of geographers have promoted the rediscovery of graph theory through the adoption of certain methods and models rejuvenated by complex networks research.

One first and key issue raised by geographers has been the relationship between network measures at node level and the more socio-economic characteristics of the connected places, such as by looking at significant statistical correlations between different centrality measures (i.e. degree, betweenness, and closeness - the latter being the inverse of the Shimbel distance) and local urban/regional data (e.g. population or Gross Domestic Product) Internet, airline, telecommunications, and scientific collaboration networks on a national or world level, as well as between street network centrality and retail and service concentration at the intra-city 
level (see Ducruet and Beauguitte, 2014 for a synthesis). The second dimension explored by geographers is the influence of distance on network topology as mentioned above, although this influence is often taken for granted by geographers One original approach has been to apply space syntax to urban streets so as to consider the network from a more cognitive point of view. Space syntax notably proposed to consider crossroads as links between streets, the latter being defined by their name or number instead of as alignments of different segments (Hillier and Hanson, 1984). Such an approach made it possible to have a completely different approach to centralities and topologies in a planar network, and to extract meaningful information on the small-world and scale-free dimension of many cities (i.e. dual graph). In the same vein, and as a third aspect of renewed graph theory applied in geography, several geographers have proposed a combination of methods rather than a strict, direct application of complex networks. It is interesting to mention that, for instance, Spatial Interaction Models (SIM) such as the gravity model (see Schintler et al., 2007), Geographic Information Systems (GIS), multiplicative growth models, and Social Network Analysis (SNA) tools, such as structural equivalence and blockmodelling, were applied, sometimes even jointly, to various networks, such as Internet backbone networks, commuting flows, road and railway networks, airline and shipping networks. This is mostly the case for regional science that was already using such methods for decades. It allowed, for instance, a better appreciation of the cost factor in network organization. A fourth dimension is the existence of subgroups of densely connected nodes in the network, often referred to as clusters or communities of nodes. Geographers were keen to detect and define such subgroups with reference to the concept of region, sometimes also mentioning the multilevel organization of networks, whereby the different subgroups are linked to each other in various ways. Methods of partitioning (or clustering) were thus applied to a number of 
networks, such as the worldwide airline network of intercity passenger flows, container shipping networks and mobile phone communication networks to verify the influence of geographic proximity in the emergence of clusters, and commuter flows between localities in order to either redefine functional urban regions or to identify secondary poles inside metropolitan areas. Such approaches were already far more advanced than the traditional single (or multiple) linkage analysis proposed in the 1960s by Nystuen and Dacey (1961) and further applied in numerous contexts afterwards. Other approaches also looked at the emergence of clusters within large cities based on the cartography of vehicle trajectories.

Fifth, geographers have attempted to go beyond the analysis of single (or simple) networks by proposing an analysis of coupled or interdependent networks of different nature, labeled multigraphs (or multiplex graphs) in the classic graph theory literature. Due to the absence of a formal mathematical definition of multigraphs, and therefore, of a precise methodology to analyze them, scholars had to create innovative ways to extract meaningful information dedicated to such graphs. Sometimes the network is simply a combination of various networks (or network of networks) in which the nature of the links is ignored, but the analysis compares the effect of adding (or removing) one or more networks at the level of nodes or the entire network. This was applied to the analysis of world regions and global cities based on various types of links (e.g. trade, diplomatic, airlines, and shipping lines). Other examples include the combination of airline and Internet networks, airline and corporate (multinational) networks, etc. Yet, geographers have poorly investigated to what extent are certain places more specialized than others in terms of combined centrality, how certain networks and nodes are more vulnerable than others according to their physical embedding and distribution across space. In particular, the local and global impacts of random or targeted attacks that are well investigated 
by physicists (e.g. cascading failures) did not yet receive much attention from geographers so far. This is rather surprising given the fact that such approaches would well apply to the analysis of cities and urban systems, which are connected by a variety of networks (e.g. power grid, water, telecommunications, transport, etc.).

Finally and as a sixth aspect of recent research based on graph theory, geographers have paid particular attention to network dynamics. Very few works have approached directly the dynamical evolution of real-world networks. Existing studies are more approaching dynamics through an analysis of successive static states of the network or phases, mostly due to a lack of continuity in available time series datasets of any kind. Other approaches to network dynamics are also those applying simulation methods to systems of cities, starting from an initial stage and testing the role of different parameters on the evolution of urban size in a relational perspective (Bretagnolle and Pumain, 2010), but often without actual information on inter-city linkages.

Graph theory has had an important influence on the way geographers studied spatial phenomena, but this influence has been stronger in the early phase of its adoption, and remained mostly true in the specific field of transportation networks. Natural scientists have been faster in adopting (and even fostering) innovations that appeared in the late 1990s through what is known as complex network research. While geographers became skeptical about the benefits of graphtheoretical methods applied to spatial issues, physicists and computer scientists had no doubt that their models, concepts and methods would improve our understanding of the uneven distribution of flows and networks, especially across space. Nevertheless, one major limitation of complex network research done by natural scientists is the absence of discussion about the link between their results and the wider social, economic, institutional and political context in which networks evolve. Stronger interactions between natural and social sciences, among which geography, is 
thus needed in order to better bridge abstract space and concrete space. In the past decades, geographers have proved to be able to explain in detail the strategies of specific actors involved in building and expanding networks of all kinds, but without a firm engagement into renewed methods of network analysis. On the contrary, natural scientists keep on refining their models and tools to analyze graphs, but without questioning the possible link with existing geographical research. Issues of scales, borders, territories, urbanization, innovation, diffusion, globalization, regional planning, environmental and socio-economic impacts are still to be explored in relation with what graph theory may provide to the concerned actors and institutions. Yet, an area well covered by geographers in the last decade, in collaboration with computer scientists, has been the search for better methods to visualize spatial networks and flows (Rozenblat and Melançon, 2013). This constitutes, perhaps, the main contribution of geography so far to graph theory and network analysis as a whole.

SEE ALSO: Network Analysis; Transport Networks; Visualization

\section{References and Further Readings}

Arlinghaus, S.L., Arlinghaus, W.C., Harary, F. (2002) Graph theory and geography: an interactive view, New York: John Wiley and Sons.

Barabási, A. L. \& Albert, R. (1999). Emergence of scaling in random networks. Science, 286(5439), 509-512.

Barthelemy, M. (2011). Spatial networks. Physics Reports, 499, 1-101.

Ducruet, C. \& Beauguitte L. (2014). Spatial science and network science: review and outcomes of a complex relationship. Networks and Spatial Economics (in press).

Ducruet, C. \& Lugo I. (2013). Structure and dynamics of transport networks: Models, methods, and applications. In J. P. Rodrigue, T. E. Notteboom and J. Shaw (Eds.), The SAGE handbook of transport studies (pp. 347-364). London: SAGE Publications Ltd. 
Haggett, P. \& Chorley, R. (1969). Network analysis in geography. London: Edward Arnold.

Hillier, B. \& Hanson, J. (1984) The social logic of space, Cambridge University Press.

Kansky, K. J. (1963). The structure of transportation networks: Relationships between network geography and regional characteristics (Research Paper No. 84). Chicago: University of Chicago.

Rozenblat, C. \& Melançon, G. (2013). Methods for multilevel analysis and visualization of geographical networks. Springer.

Schintler, L. A., Kulkarni, R., Gorman, S. \& Stough, R. (2007). Using raster-based GIS and graph theory to analyze complex networks. Networks and Spatial Economics, 7(4), 301-313.

Waters, N. (2006). Network and nodal indices. Measures of complexity and redundancy: A review. In A. Reggiani and P. Nijkamp (Eds.), Spatial dynamics, networks and modelling (pp. 13-33). Northampton: Edward Elgar Publishing.

Watts, D. J. \& Strogatz, S. H. (1998). Collective dynamics of 'small-world' networks. Nature 393(6684), 440-442.

\section{Key Words}

Networks; Spatial Analysis; Transportation 